\title{
On Length-preserving and Area-preserving Nonlocal Flow of Convex Closed Plane Curves*
}

\author{
Dong-Ho Tsai and Xiao-Liu Wang
}

July 18, 2018

\begin{abstract}
For any $\alpha>0$, we study $k^{\alpha}$-type length-preserving and area-preserving nonlocal flow of convex closed plane curves and show that these two types of flow evolve such curves into round circles in $C^{\infty}$-norm. Other relevant $k^{\alpha}$-type nonlocal flow is also discussed when $\alpha \geq 1$.
\end{abstract}

\section{Introduction.}

The curvature flow of plane curves, arising in many application fields, such as phase transitions, image processing and smoothing, etc., has received a lot of attention during the past several decades (see [ST] for example). In general, the evolution equation has the form:

$$
\left\{\begin{array}{l}
\frac{\partial X}{\partial t}(u, t)=f(k(u, t)) \mathbf{N}_{i n}(u, t), \\
X(u, 0)=X_{0}(u), \quad u \in S^{1}
\end{array}\right.
$$

where $X_{0}(u) \subset \mathbb{R}^{2}$ is a given smooth closed curve, parametrized by $u \in S^{1}$, and $X(u, t): S^{1} \times$ $[0, T) \rightarrow \mathbb{R}^{2}$ is a family of curves moving along its inward normal direction $\mathbf{N}_{i n}(u, t)$ with given speed function $f(k(u, t))$, which is a strictly increasing (parabolicity) function of the curvature $k(u, t)$ of $X(u, t)$.

When $f(k(u, t))=k(u, t)$, (1) is the famous curve shortening flow, which has been studied intensively by a great number of authors for various conditions on the initial curve $X_{0}$. One can see the book [CZ] for literature. In particular, we mention the papers [AL, Ang, G, Ga1, GH]. When $f=\frac{1}{\alpha} k^{\alpha}(\alpha \neq 0$ is a constant $)$ and $X_{0}$ is a convex ${ }^{1}$ simple closed curve, the flow (1) has been investigated in [And1, And2]. Also in [LPT1, LPT2, PT, U1, U2], they studied the case when $X_{0}$ is a locally convex non-simple closed curve. For more about (1) and its counterpart in high-dimensional space (more precisely, the mean curvature flow) one can find literature in the book [Z].

Another class of interesting curvature flow is the so-called nonlocal curvature flow, the evolution equation of which takes the form:

$$
\left\{\begin{array}{l}
X_{t}(u, t)=[F(k(u, t))-\lambda(t)] \mathbf{N}_{i n}(u, t), \\
X(u, 0)=X_{0}(u), \quad u \in S^{1},
\end{array}\right.
$$

where $X_{0}$ is a convex simple closed curve (we take its orientation to be counterclockwise), $F(k)$ is a given function of the curvature satisfying the parabolic condition $F^{\prime}(z)>0$ for all $z$ in its domain,

${ }^{*}$ AMS Subject Classifications: 53C44 (primary), 35B40, 35K15, 35K55.

${ }^{1}$ Throughout this paper, "convex" always means "uniformly convex", i.e., the curvature is strictly positive everywhere. 
and $\lambda(t)$ is a function of time which depends on certain global (nonlocal) quantities of $X(\cdot, t)$, say its length $L(t)$, enclosed area $A(t)$, or other possible global quantities like the integral of curvature over the entire curve in certain ways.

The purpose of this paper is to study $k^{\alpha}$-type nonlocal flow (2) for convex closed curves with the speed function $F(k)-\lambda(t)$ given by

$$
F(k)-\lambda(t)=k^{\alpha}-\frac{1}{2 \pi} \int_{X(\cdot, t)} k^{\alpha+1} d s, \quad \alpha>0 \quad(\mathrm{LP})
$$

or

$$
F(k)-\lambda(t)=k^{\alpha}-\frac{1}{L(t)} \int_{X(\cdot, t)} k^{\alpha} d s, \quad \alpha>0 \quad(\mathrm{AP}) .
$$

Here $s$ is the arc length parameter of $X(\cdot, t)$ and the constant $\alpha>0$ is arbitrary. The abbreviation LP (AP) indicates that the flow is "length-preserving" ("area-preserving"). We shall see shortly that under the speed function (3) the flow is length-preserving and under (41) the flow is areapreserving. Both flows have the feature that it moves the point $p \in X(\cdot, t)$ with maximal curvature inward and the point $q \in X(\cdot, t)$ with minimal curvature outward. Moreover, they both decrease the isoperimetric ratio.

We note that the above two nonlocal flows are not suitable for non-convex simple closed curves due to the curvature term $k^{\alpha}$, since it may not be defined for $k<0$. Moreover, even if $k^{\alpha}, \alpha>0$, is defined for all $k \in(-\infty, \infty)$ (say $\alpha \in \mathbb{N}$ ), the nonlocal flow for a non-convex simple closed curve $\gamma_{0}$ may easily develop self-intersections in short time. Thus we have to confine to the convex case.

When $\alpha=1$, (4) is a gradient flow of the isoperimetric deficit functional and it has been studied originally in Ga2 and recently in [CLW]. They showed that the flow preserves the convexity and enclosed area of $X_{0}$ and evolves it to a round circle in $C^{\infty}$ sense as $t \rightarrow \infty$. As far as we know, Gage's paper Ga2] seems to be the first one dealing with nonlocal flow of a convex simple closed curve. Another very recent paper is [MPW], which studied (4) for the case when $\alpha \in \mathbb{N}$ is a positive integer.

Also, when $\alpha=1$, (3) has been studied in [MZ]. They showed that the flow preserves the convexity and length of $X_{0}$ and evolves it to a round circle in $C^{\infty}$ sense as $t \rightarrow \infty$. Another interesting nonlocal flow, with $F(k)=k$, is due to [JP], who studied a gradient flow of the isoperimetric ratio functional. This gradient flow increases enclosed area and decreases length of the evolving curve. One may say that, in some sense, it is the fastest nonlocal flow (with $F(k)=k$ and among all possible choices of $\lambda(t))$ to evolve $X_{0}$ into a round circle.

Even more recently there is another interesting paper [BD], which discussed the AP nonlocal flow (with $F(k)=k$ ) for graphs (with Dirichlet boundary condition). It has application to kinetic analysis of PET (positron emission tomography) data.

We first prove the following two $C^{\infty}$ convergence results in Section 2 ,

Theorem 1.1 Assume $\alpha>0$ and $X_{0}(u), u \in S^{1}$, is a smooth convex closed curve. Then under the LP flow (3), the flow exists and preserves length for all time $t \in[0, \infty)$. Each $X(\cdot, t)$ remains smooth, convex, and it converges to a round circle with radius $L(0) / 2 \pi$ in $C^{\infty}$ topology as $t \rightarrow \infty$.

Theorem 1.2 Assume $\alpha>0$ and $X_{0}(u), u \in S^{1}$, is a smooth convex closed curve. Then under the AP flow (4), the flow exists and preserves enclosed area for all time $t \in[0, \infty)$. Each $X(\cdot, t)$ remains smooth, convex, and it converges to a round circle with radius $\sqrt{A(0) / \pi}$ in $C^{\infty}$ topology as $t \rightarrow \infty$.

Later in Section 3 we also consider other relevant $k^{\alpha}$-type nonlocal flow for $\alpha \geq 1$ (see Theorem 3.1). This flow, which is similar to [JP], has the property that it is area-increasing and lengthdecreasing. The methods of proof for Theorem 1.1 and Theorem 1.2 can be applied to Theorem 3.1 without essential changes.

\footnotetext{
${ }^{2}$ From now on, all closed curves are assumed to be simple unless otherwise stated.
} 
One can also study $1 / k^{\alpha}$-type (i.e., $\left.F(k)=-1 / k^{\alpha}\right)$ nonlocal flow for $\alpha>0$. We will not discuss it here. For the case when $F(k)=-k^{-1}$, we mention the papers [MC, $\mathrm{PY}$, [PZ]. Also see [LT] for other types of nonlocal flow.

Finally, we point out that a lot of the methods of proof used in the well-known curve shortening flow (or other related local curvature flows) are not quite applicable to the nonlocal flows we plan to discuss here. Thus we need to use different techniques.

There are also many interesting nonlocal flows in the higher dimensional case, we mention the papers $\mathrm{And} 3, \mathrm{ES}, \mathrm{H}, \mathrm{M1}, \mathrm{M} 2$.

\section{$2 \quad k^{\alpha}$-type LP and AP flow; the proof of Theorem 1.1 and Theorem 1.2.}

\subsection{Short time existence of the flow (3) (or (4)).}

The nonlocal flow (3) (or (4)) is nonlinear in nature. It is fully-nonlinear if we look at the evolution of the support function and quasilinear if we look at the evolution of the curvature. Hence one may have concern about the short time existence of a solution to these two flows. However, in view of the recent various nonlocal flow papers in the literature, it is not a problem.

More precisely, since we assume that the initial curve $X_{0}(u)$ is smooth and convex (with curvature strictly positive everywhere), the equation is strictly parabolic initially, and the nonlinear nature of the equation is not really an obstacle - for example, one can look at the treatment of short time existence in Chapter 3 of [B]. The nonlocal term does add a little complication, but it is of a very mild form and can be easily handled. See the argument in Section 7 of [M2]. Another method is to use the classical Leray-Schauder fixed point theory. See [MPW] for details.

Finally, we note that the solution to the flow (3) (or (44)) is also unique. Again, this is due to the fact that the equation is strictly parabolic initially or one can follow similar treatment as in [MPW]. Also see [ES, M1, M2].

Thus we conclude that there is a unique smooth solution to the flow (3) (or (44)) on $S^{1} \times[0, T)$ for some short time $T>0$. Moreover, as $X_{0}(u)$ is convex, by continuity, we may assume that each $X(\cdot, t)$ is also convex for $t \in[0, T)$. Later, we shall show that the convexity is preserved as long as the solution to the flow exists.

\subsection{Upper bound of the curvature; Tso's method.}

In this section, we will use Tso's method [T] to obtain a time-dependent upper bound of the curvature for $\alpha>0$.

By Section 2.1, we have a unique smooth convex solution $X(u, t)$ to both nonlocal flows on $S^{1} \times[0, T)$. According to [And1, Ang, GH] and many other papers, we can use the outward normal angle $\theta \in S^{1}=[0,2 \pi]$ to parameterize $X(\cdot, t)$. By $X(\theta, t)$ we mean the unique point on $X(\cdot, t)$ at which its outward normal $\mathbf{N}_{\text {out }}$ is given by $(\cos \theta, \sin \theta)$. With this, all evolution equations of the flow can be expressed in $(\theta, t)$ coordinates and the evolution of the curvature $k(\theta, t)$ of $X(\theta, t)$ is given by (see [GH, Ga2] for computation)

$$
\left\{\begin{array}{l}
k_{t}(\theta, t)=k^{2}(\theta, t)\left[\left(k^{\alpha}\right)_{\theta \theta}(\theta, t)+k^{\alpha}(\theta, t)-\lambda(t)\right], \\
k(\theta, 0)=k_{0}(\theta)>0, \quad(\theta, t) \in S^{1} \times[0, T),
\end{array}\right.
$$

where $k_{0}(\theta)>0$ is the initial curvature of $X_{0}(\theta)$ and

$$
\lambda(t)=\frac{1}{2 \pi} \int_{X(\cdot, t)} k^{\alpha+1} d s=\frac{1}{2 \pi} \int_{0}^{2 \pi} k^{\alpha}(\theta, t) d \theta \quad(\mathrm{LP})
$$


or

$$
\lambda(t)=\frac{1}{L(t)} \int_{X(\cdot, t)} k^{\alpha} d s=\left(\int_{0}^{2 \pi} \frac{1}{k(\theta, t)} d \theta\right)^{-1} \int_{0}^{2 \pi} k^{\alpha-1}(\theta, t) d \theta \quad(\mathrm{AP}) .
$$

Since $X_{0}(\theta)$ is a closed curve, $k_{0}(\theta)$ satisfies the integral condition

$$
\int_{0}^{2 \pi} \frac{\cos \theta}{k_{0}(\theta)} d \theta=\int_{0}^{2 \pi} \frac{\sin \theta}{k_{0}(\theta)} d \theta=0 .
$$

By (5), one can easily check that (8) is preserved under both flows.

One interesting comparison is in order. In the LP flow $\lambda(t)$ is the average of $k^{\alpha}$ over $S^{1}$ with respect to the angle $\theta \in[0,2 \pi]$, while in the AP flow $\lambda(t)$ is the average of $k^{\alpha}$ over $X(\cdot, t)$ with respect to the arc length $s \in[0, L(t)]$. Also note that, for a convex closed curve $\gamma \subset \mathbb{R}^{2}$ with curvature $k$, we have

$$
\frac{1}{L} \int_{\gamma} k^{\alpha} d s \leq \frac{1}{2 \pi} \int_{\gamma} k^{\alpha+1} d s, \quad \alpha>0
$$

due to the Hölder inequality. Thus, for the same curve, the nonlocal term in the LP flow has more outward effect than that in the AP flow.

Similar to Theorem 4.1.4 of [GH], one can verify that the nonlocal flow (3) is equivalent to the equation (5) with $\lambda(t)$ given by (6); and the nonlocal flow (4) is equivalent to the equation (5) with $\lambda(t)$ given by (7). Both have smooth initial data $k_{0}(\theta)>0$ satisfying (8). In view of this, from now on, we can just focus on the curvature equation (5) and study its long time behavior. This has become a rather standard way of studying curvature flows.

By Lemma 2.2 in Ga2, for the LP case, we have

$$
\frac{d L}{d t}(t)=0, \quad \frac{d A}{d t}(t)=-\int_{0}^{2 \pi} k^{\alpha-1}(\theta, t) d \theta+\frac{L(t)}{2 \pi} \int_{0}^{2 \pi} k^{\alpha}(\theta, t) d \theta \geq 0, \quad t \in[0, T),
$$

and for the AP case, we have

$$
\frac{d A}{d t}(t)=0, \quad \frac{d L}{d t}(t)=-\int_{0}^{2 \pi} k^{\alpha}(\theta, t) d \theta+\frac{2 \pi}{L(t)} \int_{0}^{2 \pi} k^{\alpha-1}(\theta, t) d \theta \leq 0, \quad t \in[0, T) .
$$

For $\alpha>0$, the inequalities in (10) and (11) are both due to (9) and the identity $d s=k^{-1} d \theta$.

By (10) and (11), in both flows, the evolving curve $X(\cdot, t)$ is becoming more and more circular in the sense that the isoperimetric ratio $L^{2}(t) / 4 \pi A(t)$ is decreasing in time $t \in[0, T)$. To show $C^{\infty}$ convergence of $X(\cdot, t)$, we need to do curvature estimate, which is the main work of the paper.

To go further, we need the following Bonnesen inequality for convex closed plane curves (see the book by [S], p. 324): For any convex closed curve $\gamma$ in the plane, there holds the inequality

$$
\rho L-A-\pi \rho^{2} \geq 0 \text { for all } r \leq \rho \leq R,
$$

where $r$ and $R$ are the inradius and outradius (circumradius) of $\gamma$ respectively.

By (12), we have

$$
0<\frac{L-\sqrt{L^{2}-4 \pi A}}{2 \pi} \leq r \leq R \leq \frac{L+\sqrt{L^{2}-4 \pi A}}{2 \pi},
$$

and therefore obtain the inequality

$$
1 \leq \frac{R}{r} \leq \frac{L+\sqrt{L^{2}-4 \pi A}}{L-\sqrt{L^{2}-4 \pi A}}=(\sqrt{I}+\sqrt{I-1})^{2}, \quad \text { where } \quad I=\frac{L^{2}}{4 \pi A} \geq 1 .
$$

We now can use Tso's method to prove a time-dependent upper bound of the curvature. 
Lemma 2.1 Under the LP flow (3) on $S^{1} \times[0, T)$ with $\alpha>0$, there exists a constant $C(T)>$ 0 depending on the time $T$ such that

$$
0<v(\theta, t) \leq C(T), \quad \forall(\theta, t) \in S^{1} \times[0, T)
$$

i.e.,

$$
0<k(\theta, t) \leq C(T), \quad \forall(\theta, t) \in S^{1} \times[0, T) .
$$

Here $v(\theta, t)=k^{\alpha}(\theta, t)$ and $k(\theta, t)$ is the curvature of $X(\cdot, t)$. The same result holds for the AP flow (4) on $S^{1} \times[0, T)$ with $\alpha>0$.

Proof. The evolution of $v(\theta, t)=k^{\alpha}(\theta, t)$ is given by

$$
v_{t}=\alpha v^{p}\left(v_{\theta \theta}+v-\lambda(t)\right), \quad p=1+\frac{1}{\alpha}>1, \quad(\theta, t) \in S^{1} \times[0, T),
$$

where $\lambda(t)>0$ is from (6) or (17). Since the isoperimetric ratio $I(t)=L^{2}(t) / 4 \pi A(t)$ of $X(\cdot, t)$ is decreasing in time in both flows, we have $1 \leq I(t) \leq I(0)$ for all $t \in[0, T)$. By the Bonnesen inequality (14), we have

$$
1 \leq \frac{r_{o}(t)}{r_{i}(t)} \leq(\sqrt{I(t)}+\sqrt{I(t)-1})^{2} \leq(\sqrt{I(0)}+\sqrt{I(0)-1})^{2}:=\sigma, \quad t \in[0, T)
$$

where $r_{o}(t)$ and $r_{i}(t)$ are the outradius and inradius of $X(\cdot, t)$ respectively. Hence $r_{o}(t) \leq \sigma r_{i}(t)$ and by $A(0) \leq A(t) \leq \pi r_{o}^{2}(t)$, we have $r_{i}(t) \geq \sigma^{-1} \sqrt{A(0) / \pi}$ for all $t \in[0, T)$, i.e., the inradius has time-independent positive lower bound.

Let $E(0)$ be a circle enclosed by $X_{0}$. By the maximum principle, if we shrink $E(0)$ by the $k^{\alpha_{-}}$ contraction flow (without the nonlocal term $\lambda(t)$ ), then $E(t)$ is enclosed by $X(\cdot, t)$ for all $t \in[0, T)$. The radius $r(t)$ of $E(t)$ is given by

$$
r(t)=\left[r^{1+\alpha}(0)-(1+\alpha) t\right]^{1 /(1+\alpha)}, \quad t \in\left[0, r^{1+\alpha}(0) /(1+\alpha)\right) .
$$

Now we take $E(0)$ to be an inscribed circle of $X_{0}(\cdot)$ with inradius $r(0)=r_{i}(0)$. If we use the center of $E(0)$ as the origin $O$, then the support function $u(\theta, t)$ (with respect to $O$ ) of $X(\cdot, t)$ is defined on $S^{1} \times[0, T)$ and by the above we have (since $X(\cdot, t)$ encloses $E(t)$ )

$$
u(\theta, t) \geq\left[r^{1+\alpha}(0)-(1+\alpha) t\right]^{1 /(1+\alpha)}, \quad(\theta, t) \in S^{1} \times\left[0, \min \left\{r^{1+\alpha}(0) /(1+\alpha), T\right\}\right),
$$

where now $r(0)=r_{i}(0) \geq \sigma^{-1} \sqrt{A(0) / \pi}$.

Since, in general, $T>0$ is a small time, we may assume that

$$
T \leq \frac{1}{2+2 \alpha}\left(\frac{1}{\sigma} \sqrt{\frac{A(0)}{\pi}}\right)^{1+\alpha}:=T_{1}
$$

(the case $T>T_{1}$ can be handled similarly). By (20) and $r(0) \geq \sigma^{-1} \sqrt{A(0) / \pi}$, we have $u(\theta, t) \geq$ $2 \beta$ on $S^{1} \times[0, T)$, where $\beta>0$ is a constant depending only on the initial curve $X_{0}(\cdot)$, given by

$$
\beta=\left(\frac{1}{2}\right)^{(2+\alpha) /(1+\alpha)} \frac{1}{\sigma} \sqrt{\frac{A(0)}{\pi}}, \quad \sigma=(\sqrt{I(0)}+\sqrt{I(0)-1})^{2} .
$$

Moreover, as the length $L(t)$ of $X(\cdot, t)$ is decreasing in both flows, there is a constant $C>0$ depending only on $X_{0}(\cdot)$ such that $u(\theta, t) \leq C$ on $S^{1} \times[0, T)$. Hence

$$
0<2 \beta \leq u(\theta, t) \leq C \quad \text { on } \quad S^{1} \times[0, T)
$$


Note that both $\beta$ and $C$ are independent of time.

Following [T], we consider the evolution of the quantity

$$
Q(\theta, t)=\frac{v(\theta, t)}{u(\theta, t)-\beta}, \quad v(\theta, t)=k^{\alpha}(\theta, t), \quad(\theta, t) \in S^{1} \times[0, T) .
$$

Under the nonlocal flow, the evolution of the support function is

$$
u_{t}(\theta, t)=-v(\theta, t)+\lambda(t)=-k^{\alpha}(\theta, t)+\lambda(t), \quad(\theta, t) \in S^{1} \times[0, T),
$$

where $\lambda(t)>0$ is from ([6) or (7). We compute the evolution of $Q$ on $S^{1} \times[0, T)$ to get

$$
Q_{t}=\frac{v(v-\lambda(t))}{(u-\beta)^{2}}+\frac{\alpha v^{p}\left(v_{\theta \theta}+v-\lambda(t)\right)}{u-\beta}, \quad p=1+\frac{1}{\alpha}
$$

and by

$$
Q_{\theta}=\frac{v_{\theta}}{u-\beta}-\frac{u_{\theta} v}{(u-\beta)^{2}}, \quad Q_{\theta \theta}=\frac{v_{\theta \theta}}{u-\beta}-\frac{2 u_{\theta} v_{\theta}}{(u-\beta)^{2}}+\left(\frac{2 u_{\theta}^{2}}{(u-\beta)^{3}}-\frac{u_{\theta \theta}}{(u-\beta)^{2}}\right) v
$$

we conclude

$$
\begin{aligned}
& Q_{t}=Q^{2}-\frac{\lambda(t) v}{(u-\beta)^{2}}+\alpha v^{p}\left[Q_{\theta \theta}+\frac{2 u_{\theta} v_{\theta}}{(u-\beta)^{2}}-\left(\frac{2 u_{\theta}^{2}}{(u-\beta)^{3}}-\frac{u_{\theta \theta}}{(u-\beta)^{2}}\right) v+Q-\frac{\lambda(t)}{u-\beta}\right] \\
& \leq\left\{\begin{array}{l}
\alpha v^{p} Q_{\theta \theta}+\alpha v^{p} \frac{2 u_{\theta}}{u-\beta}\left(Q_{\theta}+\frac{u_{\theta} v}{(u-\beta)^{2}}\right) \\
-\alpha v^{p+1}\left(\frac{2 u_{\theta}^{2}}{(u-\beta)^{3}}-\frac{1}{k} \frac{1}{(u-\beta)^{2}}+\frac{u}{(u-\beta)^{2}}\right)+\left(\alpha v^{p}+Q\right) Q
\end{array}\right. \\
& =\alpha v^{p} Q_{\theta \theta}+\alpha v^{p} \frac{2 u_{\theta}}{u-\beta} Q_{\theta}+Q^{2}\left[(\alpha+1)-\alpha \beta(u-\beta)^{1 / \alpha} Q^{1 / \alpha}\right] \\
& \leq \alpha v^{p} Q_{\theta \theta}+\alpha v^{p} \frac{2 u_{\theta}}{u-\beta} Q_{\theta}+Q^{2}\left[(\alpha+1)-\alpha \beta^{1+1 / \alpha} Q^{1 / \alpha}\right],
\end{aligned}
$$

where we have used the familiar identity $u_{\theta \theta}+u=1 / k$ and the inequality $u-\beta \geq \beta$ on $S^{1} \times[0, T)$ in the above. Also note that in the above estimate we have thrown away the two favorable terms containing $\lambda(t)$ due to the negative sign in front of them.

Let $Q_{0}(\alpha, \beta)=\left[2(\alpha+1) /\left(\alpha \beta^{1+1 / \alpha}\right)\right]^{\alpha}>0$. It is a constant depending only on $\alpha, \beta$. Then whenever

$$
Q_{\max }(t)=Q(\theta(t), t) \geq Q_{0}(\alpha, \beta), \quad t \in[0, T),
$$

we have

$$
\frac{d}{d t} Q_{\max }(t) \leq Q_{t}(\theta(t), t) \leq-(\alpha+1) Q_{\max }^{2}(t), \quad t \in[0, T) .
$$

Comparison principle implies

$$
Q_{\max }(t) \leq \max \left\{Q_{0}(\alpha, \beta), \frac{1}{(\alpha+1) t}\right\}, \quad \forall t \in(0, T) .
$$

In particular, we get

$$
\frac{k^{\alpha}(\theta, t)}{u(\theta, t)-\beta} \leq \max \left\{Q_{0}(\alpha, \beta), \frac{1}{(\alpha+1) t}\right\}, \quad \forall(\theta, t) \in S^{1} \times(0, T)
$$

and then by (23) we conclude

$$
k_{\max }^{\alpha}(t) \leq \max \left\{(C-\beta) Q_{0}(\alpha, \beta), \frac{C-\beta}{(\alpha+1) t}\right\}, \quad \forall t \in(0, T),
$$

which implies that near time $T$ the curvature is bounded above by a constant $C(T)$ depending on $T$, i.e., the curvature will not blow up as $t \rightarrow T$. The proof is done. 


\subsection{Lower bound of the curvature.}

We first observe the following:

Lemma 2.2 Under the LP flow (3) on $S^{1} \times[0, T)$ with $\alpha>0$, there holds the estimate

$$
\max _{S^{1} \times[0, t]} \Psi \leq \max \left\{\max _{S^{1} \times[0, t]} v^{2}, \quad \max _{S^{1} \times\{0\}} \Psi\right\}, \quad \forall t \in[0, T),
$$

where $v=k^{\alpha}$ and $\Psi=v^{2}+v_{\theta}^{2}$. In particular, we have

$$
\left|v_{\theta}(\theta, t)\right| \leq C(T), \quad \forall(\theta, t) \in S^{1} \times[0, T) .
$$

The same result holds for the AP flow (4) on $S^{1} \times[0, T)$ with $\alpha>0$.

Proof. The proof of (29) is analogous to that in Lemma I1.12 in [And1]. The evolution of $\Psi=$ $v^{2}+v_{\theta}^{2}$ is

$$
\begin{aligned}
\partial_{t} \Psi & =2 \alpha v^{p+1}\left(v_{\theta \theta}+v-\lambda(t)\right)+\left[\alpha v^{p} \Psi_{\theta \theta}-2 \alpha v^{p} v_{\theta \theta}\left(v_{\theta \theta}+v\right)\right]+\alpha p v^{p-1} v_{\theta} \Psi_{\theta}-2 \alpha p v^{p-1} v_{\theta}^{2} \lambda(t) \\
& \leq \alpha v^{p} \Psi_{\theta \theta}+\alpha p v^{p-1} v_{\theta} \Psi_{\theta}+2 \alpha v^{p+1}\left(v_{\theta \theta}+v\right)-2 \alpha v^{p} v_{\theta \theta}\left(v_{\theta \theta}+v\right), \quad p=1+\frac{1}{\alpha}>1
\end{aligned}
$$

where in the above inequality we have thrown away the terms containing $\lambda(t)$. On the time interval $[0, t]$, let $\sigma$ be the constant $\max _{S^{1} \times[0, t]} v^{2}$. Then whenever $\Psi_{\max }(s)=\Psi\left(\theta_{s}, s\right)>\sigma$ at any time $s \in[0, t]$, we have

$$
\Psi_{\theta}\left(\theta_{s}, s\right)=2 v_{\theta}\left(\theta_{s}, s\right)\left(v_{\theta \theta}\left(\theta_{s}, s\right)+v\left(\theta_{s}, s\right)\right)=0, \quad \Psi_{\theta \theta}\left(\theta_{s}, s\right) \leq 0
$$

and since $\Psi\left(\theta_{s}, s\right)>\sigma$, we must have $v_{\theta}\left(\theta_{s}, s\right) \neq 0$ and so $\left(v_{\theta \theta}\left(\theta_{s}, s\right)+v\left(\theta_{s}, s\right)\right)=0$. Now by the evolution inequality (31) we get $\partial_{t} \Psi\left(\theta_{s}, s\right) \leq 0$ at $\left(\theta_{s}, s\right)$. As a consequence of the maximum principle, we have estimate (29). Finally, the estimate (30) follows from (29) and (16).

To obtain a time-dependent lower bound of the curvature for $\alpha>0$, we use the idea from p. 64 of [MZ] (which is for the case $\alpha=1$ ). We have:

Lemma 2.3 Under the LP flow (3) on $S^{1} \times[0, T)$ with $\alpha>0$, there exists a constant $c(T)>0$ such that

$$
k(\theta, t) \geq c(T)>0, \quad \forall(\theta, t) \in S^{1} \times[0, T) .
$$

The same result holds for the AP flow (4) on $S^{1} \times[0, T)$ with $\alpha>0$.

Proof. Consider the quantity

$$
\Phi(\theta, t)=\frac{1}{k(\theta, t)}-\frac{L(t)}{2 \pi}-\frac{1}{2 \pi} \int_{0}^{t} \int_{0}^{2 \pi} k^{\alpha}(\theta, \sigma) d \theta d \sigma, \quad(\theta, t) \in S^{1} \times[0, T),
$$

where $\max _{\theta \in S^{1}} \Phi(\theta, 0) \geq 0$. In both flows, we have

$$
\begin{aligned}
\Phi_{t} & =-\left(k^{\alpha}\right)_{\theta \theta}-k^{\alpha}+\lambda(t)-\left(\lambda(t)-\frac{1}{2 \pi} \int_{0}^{2 \pi} k^{\alpha} d \theta\right)-\frac{1}{2 \pi} \int_{0}^{2 \pi} k^{\alpha}(\theta, t) d \theta \\
& =\alpha k^{\alpha+1}(\theta, t) \Phi_{\theta \theta}(\theta, t)-\alpha(\alpha+1) k^{\alpha+2}(\theta, t) \Phi_{\theta}^{2}(\theta, t)-k^{\alpha}(\theta, t) \leq \alpha k^{\alpha+1} \Phi_{\theta \theta}
\end{aligned}
$$

and the maximum principle implies

$$
\frac{1}{k(\theta, t)} \leq \max _{\theta \in S^{1}}\left(\frac{1}{k_{0}(\theta)}\right)+\frac{L(t)-L(0)}{2 \pi}+\frac{1}{2 \pi} \int_{0}^{t} \int_{0}^{2 \pi} k^{\alpha}(\theta, \sigma) d \theta d \sigma
$$

for all $(\theta, t) \in S^{1} \times[0, T)$. By (16) and the fact that $L(t)$ is decreasing, we get estimate (32). 


\subsection{Long time existence and $C^{\infty}$ convergence of the flow.}

The curvature estimates established so far and the parabolic regularity theory implies the following:

Theorem 2.4 (Long time existence of the flow.) For any $\alpha>0$, the LP flow (3) has a smooth convex solution $X(\cdot, t)$ defined for all time $t \in[0, \infty)$. Moreover, there exists a constant $C>$ 0 , independent of time, such that

$$
0<v(\theta, t) \leq C \quad \text { and } \quad\left|v_{\theta}(\theta, t)\right| \leq C, \quad \forall(\theta, t) \in S^{1} \times[0, \infty)
$$

i.e.,

$$
0<k(\theta, t) \leq C, \quad \forall(\theta, t) \in S^{1} \times[0, \infty),
$$

where $v=k^{\alpha}$. The same result holds for the AP flow (4) with $\alpha>0$.

Proof. On the domain $S^{1} \times[0, T)$, the curvature $k(\theta, t)$ has uniform positive upper and lower bounds. Parabolic regularity then implies that all space-time derivatives of $k(\theta, t)$ remain bounded on the domain $S^{1} \times[0, T)$. Therefore, the evolving curve has a smooth convex limit $X(\cdot, T)$ as $t \rightarrow T$ and one can use $X(\cdot, T)$ as a new initial curve to continue the flow. Hence the flow is defined on $S^{1} \times[0, \infty)$. It remains to explain (36).

We now have $T=\infty$ in Lemma 2.1. For any $\xi \geq 0$ we can apply Tso's argument on the interval $\left[\xi, \xi+T_{1}\right]\left(T_{1}\right.$ is from (21), which is independent of $\left.\xi\right)$. The support function $u$ is now with respect to the center of an inscribed circle of $X(\cdot, \xi)$ with radius $r_{i}(\xi) \geq \sigma^{-1} \sqrt{A(0) / \pi}$. The constants $C$ and $\beta$ are the same as before (both are independent of $\xi$ ) and we still have inequality (23) on $S^{1} \times\left[\xi, \xi+T_{1}\right]$. Since the time interval is $\left[\xi, \xi+T_{1}\right]$, the estimate (27) now becomes

$$
Q_{\max }(t) \leq \max \left\{Q_{0}(\alpha, \beta), \frac{1}{(\alpha+1)(t-\xi)}\right\}, \quad \forall t \in\left(\xi, \xi+T_{1}\right)
$$

and we get

$$
0<k_{\max }^{\alpha}(t) \leq \max \left\{(C-\beta) Q_{0}(\alpha, \beta), \frac{C-\beta}{(\alpha+1)(t-\xi)}\right\}, \quad \forall t \in\left(\xi, \xi+T_{1}\right) .
$$

In particular, we get

$$
0<k_{\max }^{\alpha}\left(\xi+T_{1}\right) \leq \max \left\{(C-\beta) Q_{0}(\alpha, \beta), \frac{C-\beta}{(\alpha+1) T_{1}}\right\} .
$$

As $\xi \geq 0$ is arbitrary, the first estimate in (36) is confirmed. The second estimate follows from (29) in Lemma 2.2.

Until now, we have not yet proved a time-independent positive lower bound of the curvature. To prove it, we consider the case $0<\alpha<1$ and $\alpha>1$ separately.

\subsubsection{The case $0<\alpha<1$.}

For the case $0<\alpha<1$, we can improve Lemma 2.3 as:

Lemma 2.5 Under the LP flow (3) on $S^{1} \times[0, \infty)$ with $0<\alpha<1$, there exists a constant $c>$ 0 , independent of time, such that

$$
k(\theta, t) \geq c>0, \quad \forall(\theta, t) \in S^{1} \times[0, \infty) .
$$

The same result holds for the AP flow (4) on $S^{1} \times[0, \infty)$ with $0<\alpha<1$. 
Proof. We use the idea from p. 349 of [And1]. In view of the relation $k=d \theta / d s$, the gradient estimate in (36) can be rewritten as $\left|\left(k^{\alpha-1}(\cdot, t)\right)_{s}\right| \leq C$ for some different constant $C$ independent of time, where $s$ is the arc length parameter of $X(\cdot, t)$. Using this and the mean value theorem, together with the property that the length of $X(\cdot, t)$ is decreasing in time in both flows, we have

$$
\left|\frac{1}{k^{1-\alpha}\left(s_{2}, t\right)}-\frac{1}{k^{1-\alpha}\left(s_{1}, t\right)}\right| \leq C\left|s_{2}-s_{1}\right| \leq C, \quad 0<\alpha<1
$$

for all $t \in[0, \infty)$ and all $s_{1}, s_{2}$ on the curve $X(\cdot, t)$. On the other hand, for each $t \in[0, \infty)$, there exists some $\theta(t) \in S^{1}$ such that

$$
L(t)=\int_{0}^{2 \pi} \frac{1}{k(\theta, t)} d \theta=\frac{2 \pi}{k(\theta(t), t)} \leq L(0) .
$$

This implies, at each time, the existence of some value of $s(t)$ such that

$$
0<\frac{1}{k^{1-\alpha}(s(t), t)} \leq\left(\frac{L(0)}{2 \pi}\right)^{1-\alpha}, \quad 0<\alpha<1 .
$$

The result follows by (41) and (40).

For the case $0<\alpha<1$, Theorem 2.4 and Lemma 2.5, together with parabolic regularity theory, imply that both flows are uniformly parabolic on $S^{1} \times[0, \infty)$ and the evolving curve $X(\cdot, t)$ converges as $t \rightarrow \infty$ in $C^{\infty}\left(S^{1}\right)$ to a circle with radius $L(0) / 2 \pi$ (in (3) ) or $\sqrt{A(0) / \pi}($ in (44)). In fact, due to the uniform positive upper and lower bounds of the curvature, Lemmas 2.6, 2.9, 2.10, Theorem 2.11 and Remark 2.12 in the next section are all valid for the case $0<\alpha<1$.

Our conclusion is that now Theorem 1.1 and Theorem 1.2 have been proved for the case $0<$ $\alpha<1$.

\subsubsection{The case $\alpha>1$.}

It remains to prove a time-independent positive lower bound of the curvature for the case $\alpha>1$. Note that the double integral in (35) may tend to infinity as $t \rightarrow \infty$. Therefore, one needs to do more to exclude the possibility that the lower bound may tend to zero as $t \rightarrow \infty$.

We have:

Lemma 2.6 Under the LP flow (3) on $S^{1} \times[0, \infty)$ with $\alpha>1$, we have

$$
\frac{d A}{d t}(t) \rightarrow 0 \quad \text { as } \quad t \rightarrow \infty
$$

and under the AP flow (4) on $S^{1} \times[0, \infty)$ with $\alpha>1$, we have

$$
\frac{d L}{d t}(t) \rightarrow 0 \quad \text { as } \quad t \rightarrow \infty
$$

Proof. Assume not for (42). Since $A^{\prime}(t) \geq 0$, there exists a constant $C_{0}>0$ independent of time and a sequence of times $\left\{t_{i}\right\}_{i=1}^{\infty}$ going to infinity such that $A^{\prime}\left(t_{i}\right) \geq C_{0}>0$ for all $i=1,2,3, \ldots$ Also note that

$$
\begin{aligned}
A^{\prime \prime}(t) & =\frac{d}{d t}\left[-\int_{0}^{2 \pi} k^{\alpha-1}(\theta, t) d \theta+\frac{L(t)}{2 \pi} \int_{0}^{2 \pi} k^{\alpha}(\theta, t) d \theta\right], \quad L(t)=L(0) \\
& =-\int_{0}^{2 \pi}(\alpha-1) v\left(v_{\theta \theta}+v-\lambda(t)\right) d \theta+\frac{L(0)}{2 \pi} \int_{0}^{2 \pi} \alpha v^{p}\left(v_{\theta \theta}+v-\lambda(t)\right) d \theta \\
& :=I(t)+I I(t),
\end{aligned}
$$


where $p=1+1 / \alpha$. By (36) $)$, we have

$$
|I(t)|=\left|-(\alpha-1) \int_{0}^{2 \pi}\left(-v_{\theta}^{2}+v^{2}-v \lambda(t)\right) d \theta\right| \leq C_{1}, \quad \lambda(t)=\frac{1}{2 \pi} \int_{0}^{2 \pi} k^{\alpha}(\theta, t) d \theta
$$

for some constant $C_{1}$ independent of time. Also

$$
|I I(t)|=\left|\frac{L(0)}{2 \pi} \int_{0}^{2 \pi}\left(-\alpha p v^{p-1} v_{\theta}^{2}+\alpha v^{p+1}-\alpha v^{p} \lambda(t)\right) d \theta\right| \leq C_{2}, \quad p=1+\frac{1}{\alpha}>1
$$

for another constant $C_{2}$ independent of time. Therefore we obtain $\left|A^{\prime \prime}(t)\right| \leq C_{3}$ for all $t \in[0, \infty)$ for some constant $C_{3}$ independent of time. As the derivative of $A^{\prime}(t)$ is uniformly bounded, one can find a number $\rho_{0}$ independent of $t_{i}$ such that

$$
A^{\prime}(t) \geq \frac{C_{0}}{2}>0, \quad \forall t \in\left[t_{i}, t_{i}+\rho_{0}\right], \quad i=1,2,3, \ldots
$$

This implies that $A(\infty)-A(0)=\int_{0}^{\infty} A^{\prime}(t) d t=\infty$, contradicting to the inequality $0<4 \pi A(t) \leq$ $L^{2}(t)=L^{2}(0)$ for all time. The proof of (42) is done.

Similarly, assume not for (43). Since $L^{\prime}(t) \leq 0$, there exists a constant $C_{0}>0$ and a sequence of times $\left\{t_{i}\right\}_{i=1}^{\infty}$ going to infinity such that $L^{\prime}\left(t_{i}\right) \leq-C_{0}<0$ for all $i=1,2,3$, ... Also note that

$$
\begin{aligned}
L^{\prime \prime}(t) & =\frac{d}{d t}\left[-\int_{0}^{2 \pi} k^{\alpha} d \theta+\frac{2 \pi}{L(t)} \int_{0}^{2 \pi} k^{\alpha-1} d \theta\right] \\
& =\left\{\begin{array}{l}
-\int_{0}^{2 \pi} \alpha v^{p}\left(v_{\theta \theta}+v-\lambda(t)\right) d \theta+\frac{2 \pi}{L(t)} \int_{0}^{2 \pi}(\alpha-1) v\left(v_{\theta \theta}+v-\lambda(t)\right) d \theta \\
-\frac{2 \pi}{L^{2}(t)}\left(-\int_{0}^{2 \pi} k^{\alpha} d \theta+\frac{2 \pi}{L(t)} \int_{0}^{2 \pi} k^{\alpha-1} d \theta\right) \int_{0}^{2 \pi} k^{\alpha-1} d \theta, \quad \alpha>1,
\end{array}\right.
\end{aligned}
$$

where $\lambda(t)=L^{-1}(t) \int_{0}^{2 \pi} k^{\alpha-1}(\theta, t) d \theta, \alpha>1$. By (36) again, $\lambda(t)$ is uniformly bounded and we have $\left|L^{\prime \prime}(t)\right| \leq C_{4}$ for all $t \in[0, \infty)$ for some constant $C_{4}$ independent of time. As the derivative of $L^{\prime}(t)$ is uniformly bounded, one can find a number $\rho_{0}$ independent of $t_{i}$ such that

$$
L^{\prime}(t) \leq-\frac{C_{0}}{2}<0, \quad \forall t \in\left[t_{i}, t_{i}+\rho_{0}\right], \quad i=1,2,3, \ldots
$$

This implies that $L(\infty)-L(0)=\int_{0}^{\infty} L^{\prime}(t) d t=-\infty$, contradicting to the isoperimetric inequality $L^{2}(t) \geq 4 \pi A(t)=4 \pi A(0)$ for all time. The proof is done.

To go further we recall the following inequality in Andrews And1:

Lemma 2.7 Let $M$ be a compact Riemannian manifold with a volume form $d \mu$, and let $\xi$ be a continuous function on $M$. Then for any decreasing continuous function $F: \mathbb{R} \rightarrow \mathbb{R}$, we have

$$
\int_{M} \xi d \mu \int_{M} F(\xi) d \mu \geq \int_{M} d \mu \int_{M} \xi F(\xi) d \mu
$$

If $F$ is strictly decreasing, then equality holds if and only if $\xi$ is a constant function on $M$.

Remark 2.8 If $F$ is increasing, then we have $\leq$ in (47).

Next, we prove the following: 
Lemma 2.9 Under the LP flow (3) on $S^{1} \times[0, \infty)$ with $\alpha>1$, we have

$$
\lim _{t \rightarrow \infty}\left\|k(\cdot, t)-\frac{2 \pi}{L(0)}\right\|_{C^{0}\left(S^{1}\right)}=0
$$

Proof. Assume not. Then there exists a sequence of times $\left\{t_{i}\right\}_{i=1}^{\infty}$ going to infinity such that

$$
\left\|k\left(\cdot, t_{i}\right)-\frac{2 \pi}{L(0)}\right\|_{C^{0}\left(S^{1}\right)} \geq \varepsilon>0, \quad \forall i
$$

for some $\varepsilon>0$. As $v$ and $v_{\theta}$ are both uniformly bounded, there is a subsequence of $\left\{t_{i}\right\}_{i=1}^{\infty}$, still denoted as $\left\{t_{i}\right\}_{i=1}^{\infty}$, such that $v\left(\theta, t_{i}\right)=k^{\alpha}\left(\theta, t_{i}\right)$ converges uniformly on $S^{1}$ to a Lipschitz continuous function $w(\theta) \geq 0$ as $i \rightarrow \infty$. In particular, since $\alpha>1$, both $k\left(\theta, t_{i}\right)$ and $k^{\alpha-1}\left(\theta, t_{i}\right)$ converge uniformly to $w^{1 / \alpha}(\theta)$ and $w^{1-1 / \alpha}(\theta)$ respectively. At this moment, we can not guarantee that $w^{1 / \alpha}(\theta) \geq 0$ is strictly positive. Hence we cannot conclude that the integral of $1 / k\left(\theta, t_{i}\right)$ converges to the integral of $w^{-1 / \alpha}(\theta)$. One needs to do more. By (42), we have

$$
0=\lim _{i \rightarrow \infty} \frac{d A}{d t}\left(t_{i}\right)=-\int_{0}^{2 \pi} w^{1-\frac{1}{\alpha}}(\theta) d \theta+\frac{L(0)}{2 \pi} \int_{0}^{2 \pi} w(\theta) d \theta, \quad 1-\frac{1}{\alpha}>0 .
$$

Also note that

$$
L(0)=\int_{0}^{2 \pi} \frac{1}{k\left(\theta, t_{i}\right)} d \theta, \quad \forall i
$$

and by Fatou's lemma, we have

$$
0 \leq \int_{0}^{2 \pi} \frac{1}{w^{1 / \alpha}(\theta)} d \theta \leq L(0)
$$

Thus the Lebesgue integral $\int_{0}^{2 \pi} w^{-1 / \alpha}(\theta) d \theta$ converges, i.e., $w^{1 / \alpha}(\theta)>0$ almost everywhere on $S^{1}$. Now by (50) and (51), we have

$$
2 \pi \int_{0}^{2 \pi} w^{1-\frac{1}{\alpha}}(\theta) d \theta \geq \int_{0}^{2 \pi} \frac{1}{w^{1 / \alpha}(\theta)} d \theta \int_{0}^{2 \pi} w(\theta) d \theta .
$$

On the other hand, by inequality (47), we also have (take $\left.F(\xi)=1 /\left(\xi^{1 / \alpha}+\varepsilon\right), \xi \geq 0, \varepsilon>0\right)$

$$
\int_{0}^{2 \pi} w(\theta) d \theta \int_{0}^{2 \pi} \frac{1}{w^{1 / \alpha}(\theta)+\varepsilon} d \theta \geq 2 \pi \int_{0}^{2 \pi} \frac{w(\theta)}{w^{1 / \alpha}(\theta)+\varepsilon} d \theta, \quad \forall \varepsilon>0 .
$$

Letting the constant $\varepsilon \rightarrow 0^{+}$, we obtain

$$
\int_{0}^{2 \pi} w(\theta) d \theta \int_{0}^{2 \pi} \frac{1}{w^{1 / \alpha}(\theta)} d \theta \geq 2 \pi \int_{0}^{2 \pi} w^{1-\frac{1}{\alpha}}(\theta) d \theta
$$

Combine (52) and (153) to get

$$
2 \pi \int_{0}^{2 \pi} w^{1-\frac{1}{\alpha}}(\theta) d \theta=\int_{0}^{2 \pi} \frac{1}{w^{1 / \alpha}(\theta)} d \theta \int_{0}^{2 \pi} w(\theta) d \theta
$$

i.e., the following iterated integral vanishes

$$
\frac{1}{2} \int_{0}^{2 \pi} \int_{0}^{2 \pi}\left[\left(\frac{1}{w^{1 / \alpha}(x)}-\frac{1}{w^{1 / \alpha}(y)}\right)(w(x)-w(y))\right] d x d y=0 .
$$

As the integrand in (54) is nonpositive almost everywhere on $S^{1} \times S^{1}$ and $w(\theta) \geq 0$ is a continuous function, we must have $w(\theta)=C$ everywhere on $S^{1}$, where $C$ is the constant given by $(2 \pi / L(0))^{\alpha}$ due to (50). This implies that $k\left(\cdot, t_{i}\right)$ converges uniformly to the constant $2 \pi / L(0)$ as $i \rightarrow \infty$, contradicting to (49). The proof is done.

Finally, we have: 
Lemma 2.10 Under the AP flow (4) on $S^{1} \times[0, \infty)$ with $\alpha>1$, we have

$$
\lim _{t \rightarrow \infty}\left\|k(\cdot, t)-\sqrt{\frac{\pi}{A(0)}}\right\|_{C^{0}\left(S^{1}\right)}=0
$$

Proof. The proof is also identical to Lemma 2.9, We now have

$$
0=\lim _{i \rightarrow \infty} \frac{d L}{d t}\left(t_{i}\right)=-\int_{0}^{2 \pi} w(\theta) d \theta+\frac{2 \pi}{L(\infty)} \int_{0}^{2 \pi} w^{1-\frac{1}{\alpha}}(\theta) d \theta, \quad 1-\frac{1}{\alpha}>0 .
$$

where $L(\infty)=\lim _{t \rightarrow \infty} L(t)$ and by Fatou's lemma, we have (51) and so we arrive at the inequality (52) again. The rest of the proof goes the same as in Lemma 2.9 and we conclude that $k(\cdot, t)$ converges uniformly to the constant $2 \pi / L(\infty)$ as $t \rightarrow \infty$. Since the limit is a circle, we have $2 \pi / L(\infty)=\sqrt{\pi / A(\infty)}=\sqrt{\pi / A(0)}$. The proof is done.

Now by Lemma 2.9 and Lemma 2.10 we can obtain the following $C^{\infty}$ convergence of curvature:

Theorem 2.11 Under the LP flow (3) with $\alpha>1$ we have

$$
\lim _{t \rightarrow \infty}\left\|k(\cdot, t)-\frac{2 \pi}{L(0)}\right\|_{C^{m}\left(S^{1}\right)}=0, \quad \forall m=0,1,2,3, \ldots
$$

and under the AP flow (4) with $\alpha>1$ we have

$$
\lim _{t \rightarrow \infty}\left\|k(\cdot, t)-\sqrt{\frac{\pi}{A(0)}}\right\|_{C^{m}\left(S^{1}\right)}=0, \quad \forall m=0,1,2,3, \ldots
$$

Proof. By (48), the curvature equation in (5) is uniformly parabolic and the parabolic regularity theory implies that all space-time derivatives of $k(\theta, t)$ are uniformly bounded by constants depending only on the order of differentiation. By induction and the Arzela-Ascoli theorem, we can easily obtain (57) and (158).

The proof of Theorem 1.1 and Theorem 1.2 is now complete.

Remark 2.12 Strictly speaking, the proof of Theorem 1.1 and Theorem 1.2 is not quite complete yet because in Theorem 2.11 it is only shown that, in both flows, the curvature tends to a constant eventually. Conceivably the evolving curve $X(\cdot, t)$ may escape to infinity or oscillate indefinitely. ${ }^{3}$. To show that this will not happen, one needs additional arguments to ensure that the flow does tend to a unique fixed circle eventually. We only give a brief description here. The idea is to prove that the curvature $k(\cdot, t)$ converges to a constant in an exponentially-decay way, like what has been done in Gage-Hamilton [GH]. One can also see Theorem 5.10. in the recent paper [MPW], which has successfully generalized Gage-Hamilton's argument to the nonlocal flow (4) for the case $\alpha \in \mathbb{N}$. Recall that the function $v=k^{\alpha}$ satisfies the equation

$$
v_{t}(\theta, t)=\alpha v^{p}(\theta, t)\left[v_{\theta \theta}(\theta, t)+v(\theta, t)-\lambda(t)\right], \quad p=1+\frac{1}{\alpha}>1, \quad(\theta, t) \in S^{1} \times[0, \infty)
$$

and we have know that $v(\theta, t)$ tends to a positive constant as $t \rightarrow \infty$ in the space $C^{\infty}\left(S^{1}\right)$ and $\lambda(t)$ also tends to a positive constant as $t \rightarrow \infty$ (with all of its derivatives tend to zero as $t \rightarrow \infty$ ). With this, plus a further analysis (for example, linearization) of the equation (59), can convince us that the difference $v(\theta, t)-\lambda(t)$ does decay to zero exponentially in $C^{\infty}\left(S^{1}\right)$ (one can also mimic the method in [MPW] or see [ES]). Next, by the equation of the support function, which is (see (25))

$$
u_{t}(\theta, t)=-v(\theta, t)+\lambda(t)=-k^{\alpha}(\theta, t)+\lambda(t), \quad(\theta, t) \in S^{1} \times[0, \infty),
$$

\footnotetext{
${ }^{3}$ We thank the referee for pointing out this important issue, which we had neglected at first.
} 
we know $u_{t}(\theta, t)$ also decays to zero exponentially in $C^{\infty}\left(S^{1}\right)$. By the identity $u_{\theta \theta}+u=1 / k$, one can express $u$ as

$$
u(\theta, t)=a(t) \cos \theta+b(t) \sin \theta+\int_{0}^{\theta} \frac{1}{k(\sigma, t)} \sin (\theta-\sigma) d \sigma, \quad(\theta, t) \in S^{1} \times[0, \infty),
$$

where $a(t)=u(0, t)$ and $b(t)=u_{\theta}(0, t)$. By (61), we get

$$
u_{\theta}(\theta, t)=-a(t) \sin \theta+b(t) \cos \theta+\int_{0}^{\theta} \frac{1}{k(\sigma, t)} \cos (\theta-\sigma) d \sigma
$$

and then

$$
u_{t}(0, t)=a^{\prime}(t), \quad\left(u_{\theta}\right)_{t}(0, t)=\left(u_{t}\right)_{\theta}(0, t)=b^{\prime}(t), \quad t \in[0, \infty) .
$$

Since $a^{\prime}(t)$ and $b^{\prime}(t)$ both decay to zero exponentially, the two integrals $\int_{0}^{\infty} a^{\prime}(t) d t$ and $\int_{0}^{\infty} b^{\prime}(t) d t$ both converge. By

$$
\lim _{t \rightarrow \infty} a(t)=a(0)+\int_{0}^{\infty} a^{\prime}(t) d t, \quad \lim _{t \rightarrow \infty} b(t)=b(0)+\int_{0}^{\infty} b^{\prime}(t) d t
$$

and (61), we conclude that the support function u has a limit:

$$
\lim _{t \rightarrow \infty} u(\theta, t)=a \cos \theta+b \sin \theta+c, \quad \forall \theta \in S^{1},
$$

for some constants $a, b, c \in \mathbb{R}$ with $c>0$. Thus the circle in Theorem 1.1 is a fixed circle centered at $(a, b) \in \mathbb{R}^{2}$ with radius $L(0) / 2 \pi$ and the circle in Theorem 1.2 is a fixed circle centered at $(a, b) \in \mathbb{R}^{2}$ with radius $\sqrt{A(0) / \pi}$.

\section{Other $k^{\alpha}$-type nonlocal flow for $\alpha \geq 1$.}

When $\alpha \geq 1$, using certain geometric inequalities for convex closed plane curves, we can consider two additional types of nonlocal flow. Both flows have the interesting feature that $L(t)$ is decreasing and $A(t)$ is increasing.

Recall the following two inequalities for convex closed plane curves (note that (63) is the same as (9)):

$$
\int_{0}^{2 \pi} k^{\beta-1}(\theta) d \theta \leq \frac{L}{2 \pi} \int_{0}^{2 \pi} k^{\beta}(\theta) d \theta, \quad \forall \beta \geq 0
$$

and

$$
\int_{0}^{2 \pi} k^{\beta}(\theta) d \theta \leq \frac{2 A}{L} \int_{0}^{2 \pi} k^{\beta+1}(\theta) d \theta, \quad \forall \beta \geq 0,
$$

where for $\beta>0$ the equality holds in (63) if and only if $\gamma$ is a circle; and for $\beta \geq 0$ the equality holds in (64) if and only if $\gamma$ is a circle. When $\beta=0$, we have equality in (63) for any convex closed curve; and when $\beta=0$, (64) becomes

$$
\int_{0}^{2 \pi} k(\theta) d \theta \geq \frac{\pi L}{A}
$$

which is precisely Gage's isoperimetric inequality for convex closed curves (see [Ga3]). In fact, (64) is a combination of Gage's inequality and Hölder inequality (or Andrews's inequality (47)). One can also see Corollary 1.10. in [LT].

Combined with (64) and the inequality $L^{2} \geq 4 \pi A$, for $\alpha \geq 1$ we have the following two inequalities for any convex closed plane curve:

$$
\frac{1}{L} \int_{0}^{2 \pi} k^{\alpha-1} d \theta \leq \frac{2 A}{L^{2}} \int_{0}^{2 \pi} k^{\alpha} d \theta \leq \frac{1}{2 \pi} \int_{0}^{2 \pi} k^{\alpha} d \theta, \quad \alpha \geq 1
$$


or

$$
\frac{1}{L} \int_{0}^{2 \pi} k^{\alpha-1} d \theta \leq \frac{L}{4 \pi A} \int_{0}^{2 \pi} k^{\alpha-1} d \theta \leq \frac{1}{2 \pi} \int_{0}^{2 \pi} k^{\alpha} d \theta, \quad \alpha \geq 1 .
$$

Motivated by (66) and (67), we can prove the following:

Theorem 3.1 Assume $\alpha \geq 1$ and $X_{0}(u), u \in S^{1}$, is a smooth convex closed curve. Then the nonlocal flow (2), where

$$
F(k)=k^{\alpha}, \quad \lambda(t)=\frac{2 A(t)}{L^{2}(t)} \int_{X(\cdot, t)} k^{\alpha+1} d s
$$

has a smooth convex solution for all time $t \in[0, \infty)$. Moreover, it converges to a round circle in $C^{\infty}$ topology as $t \rightarrow \infty$. The same result holds if

$$
F(k)=k^{\alpha}, \quad \lambda(t)=\frac{L(t)}{4 \pi A(t)} \int_{X(\cdot, t)} k^{\alpha} d s .
$$

Remark 3.2 When $\alpha=1, \lambda(t)$ in (69) becomes $\lambda=L / 2 A$, which is a gradient flow of the isoperimetric ratio functional. It has been studied in [JP].

Remark 3.3 By (66), one can view (68) as a flow below the LP case (hence $L(t)$ is decreasing) and above the the $A P$ case (hence $A(t)$ is increasing). The same for (69).

Proof. Again, the flow has a unique smooth convex solution for short time $[0, T)$ and we can use the outward normal angle $\theta$ as a parameter. Now for the flow (2) with $F(k)$ and $\lambda(t)$ given by (68) or (69), we have

$$
\frac{d L}{d t}=2 \pi \lambda(t)-\int_{0}^{2 \pi} k^{\alpha}(\theta, t) d \theta \leq 0, \quad t \in[0, T)
$$

and

$$
\frac{d A}{d t}=\lambda(t) L(t)-\int_{0}^{2 \pi} k^{\alpha-1}(\theta, t) d \theta \geq 0, \quad t \in[0, T) .
$$

Thus $L(t)$ is decreasing and $A(t)$ is increasing. In particular, the isoperimetric ratio $L^{2}(t) / 4 \pi A(t)$ is decreasing.

Next, we claim that all of the previous curvature estimates are valid for $\lambda(t)$ given by (68) or (69). First, the proof in Lemma 2.1 (i.e., Tso's estimate) carries over to this case since now the length is decreasing and the area is increasing (in both (68) and (69)). Moreover, the inequality (26) is valid as long as $\lambda(t)$ is a positive quantity. Similarly, Lemma 2.2 is also valid as long as $\lambda(t)$ is positive (see Remark 2.12). In the proof of inequality (34), the term $\lambda(t)$ has been cancelled. Hence Lemma 2.3 is also valid here. Consequently, we have Theorem 2.4 and the flow is smooth, convex, defined on $S^{1} \times[0, \infty)$.

Next we check the validity of Lemma 2.6. For $\lambda(t)$ given by (68) we have

$$
\lambda^{\prime}(t)=\frac{2 A(t)}{L^{2}(t)} \int_{0}^{2 \pi} \alpha v^{p}\left(v_{\theta \theta}+v-\lambda(t)\right) d \theta+\left(\int_{0}^{2 \pi} k^{\alpha} d \theta\right) \frac{d}{d t}\left(\frac{2 A(t)}{L^{2}(t)}\right), \quad p=1+\frac{1}{\alpha},
$$

and, same as before, it is easy to see that $|\lambda(t)| \leq C$ and $\left|\lambda^{\prime}(t)\right| \leq C$ for all $t \in[0, \infty)$ for some constant $C$ independent of time. Hence we have

$$
\left|\frac{d^{2} A}{d t^{2}}(t)\right|=\left|\lambda^{\prime}(t) L(t)+\lambda(t) L^{\prime}(t)-\int_{0}^{2 \pi}(\alpha-1) v\left(v_{\theta \theta}+v-\lambda(t)\right) d \theta\right| \leq C
$$

and

$$
\left|\frac{d^{2} L}{d t^{2}}(t)\right|=\left|2 \pi \lambda^{\prime}(t)-\int_{0}^{2 \pi} \alpha v^{p}\left(v_{\theta \theta}+v-\lambda(t)\right) d \theta\right| \leq C
$$


for all $t \in[0, \infty)$ for some constant $C$ independent of time. The above two estimates imply

$$
\frac{d A}{d t}(t)=\lambda(t) L(t)-\int_{0}^{2 \pi} k^{\alpha-1}(\theta, t) d \theta \rightarrow 0 \quad \text { as } \quad t \rightarrow \infty
$$

and

$$
\frac{d L}{d t}(t)=2 \pi \lambda(t)-\int_{0}^{2 \pi} k^{\alpha}(\theta, t) d \theta \rightarrow 0 \quad \text { as } \quad t \rightarrow \infty .
$$

Thus Lemma 2.6 is valid for $\lambda(t)$ given by (68). One can check that Lemma 2.6 is also valid for $\lambda(t)$ given by (69),

Finally, it remains to check the $C^{0}$ convergence of the curvature. For $\lambda(t)$ given by (68), since we have both (72) and (73), (50) in Lemma 2.9 can be replaced by

$$
0=\lim _{i \rightarrow \infty} \frac{d L}{d t}\left(t_{i}\right)=\frac{4 \pi A(\infty)}{L^{2}(\infty)} \int_{0}^{2 \pi} w(\theta) d \theta-\int_{0}^{2 \pi} w(\theta) d \theta
$$

and

$$
0=\lim _{i \rightarrow \infty} \frac{d A}{d t}\left(t_{i}\right)=\frac{2 A(\infty)}{L(\infty)} \int_{0}^{2 \pi} w(\theta) d \theta-\int_{0}^{2 \pi} w^{1-\frac{1}{\alpha}}(\theta) d \theta .
$$

Hence $L^{2}(\infty)=4 \pi A(\infty)$ and $(75)$ becomes

$$
\frac{L(\infty)}{2 \pi} \int_{0}^{2 \pi} w(\theta) d \theta=\int_{0}^{2 \pi} w^{1-\frac{1}{\alpha}}(\theta) d \theta
$$

By Fatou's lemma again, we get

$$
0 \leq \int_{0}^{2 \pi} \frac{1}{w^{1 / \alpha}(\theta)} d \theta \leq L(\infty)
$$

and conclude the same inequality as (52). Same as in the proof of Lemma 2.9 we have $w(\theta)=$ $C$ everywhere on $S^{1}$ for some constant $C$, where $C>0$ is the constant given by $(2 \pi / L(\infty))^{\alpha}$. This implies that $k\left(\cdot, t_{i}\right)$ converges uniformly to the constant $2 \pi / L(\infty)$ as $i \rightarrow \infty$. The proof for the case (69) is similar.

Until now, we have obtained a $C^{0}$ convergence of the curvature of the flow for $\lambda(t)$ given by (68) or (69). The $C^{\infty}$ convergence of the flow follows from standard regularity theory. The proof of Theorem 3.1 is finished (see Remark 2.12 also).

\section{Appendix; entropy estimate.}

Results in this short appendix section are supplementary. They are interesting on their own. The property that we will claim is the so-called "entropy estimate" (we take this terminology from And1, p. 322). It is about the monotonicity of certain integral quantities.

We need the following inequality, which is a consequence of the Minkowski inequality (1.6) in p. 322 of [And1]:

Lemma 4.1 Let $\gamma$ be a convex closed curve with curvature $k(\theta)>0$, where $\theta$ is its outward normal angle. Then for any $C^{2}$ function $\Phi:(0, \infty) \rightarrow \mathbb{R}$, we have the following inequalities:

$$
2 \pi \int_{0}^{2 \pi} \Phi(k)\left[\Phi(k)_{\theta \theta}+\Phi(k)\right] d \theta \leq\left(\int_{0}^{2 \pi} \Phi(k) d \theta\right)^{2}
$$

and

$$
2 A \int_{0}^{2 \pi} \Phi(k)\left[\Phi(k)_{\theta \theta}+\Phi(k)\right] d \theta \leq\left(\int_{0}^{2 \pi} \frac{\Phi(k)}{k} d \theta\right)^{2},
$$

where $\Phi(k)=\Phi(k(\theta))$ and $A$ is the enclosed area of $\gamma$. 
Proof. In p. 322 of And1, for a convex closed plane curve $\gamma$ with support function $u(\theta)$ and curvature $k(\theta)$, we can take $s_{1}(\theta)=u(\theta)$ and $s_{2}(\theta)=\Phi(k(\theta))$ in the Minkowski inequality, where $\Phi(z)$ is an arbitrary $C^{2}$ function on $(0, \infty)$. This proves (77). On the other hand, one can also take $s_{1}(\theta)=1$ and $s_{2}(\theta)=\Phi(k(\theta))$. Then we get (176) $)$.

If we consider the flow $X_{t}=\Phi(k) \mathbf{N}_{i n}$, then the length $L(t)$ and enclosed area $A(t)$ of $X(\cdot, t)$ will satisfy

$$
\frac{d A}{d t}(t)=-\int_{0}^{2 \pi} \frac{\Phi(k(\theta, t))}{k(\theta, t)} d \theta, \quad \frac{d L}{d t}(t)=-\int_{0}^{2 \pi} \Phi(k(\theta, t)) d \theta .
$$

In particular, when $\Phi(k)=F(k)-\lambda(t)$ is from the LP flow (3) or from the AP flow (4), the right hand sides of (76) and (77) will become zero, which gives a useful estimate. More precisely, we have:

Lemma 4.2 (Entropy estimate.) Under the LP flow (3), we have

$$
\text { if } 0<\alpha<1, \quad \int_{0}^{2 \pi} k^{\alpha-1}(\theta, t) d \theta \text { is increasing in time } t \in[0, \infty) \text {, }
$$

and

$$
\text { if } \alpha>1, \quad \int_{0}^{2 \pi} k^{\alpha-1}(\theta, t) d \theta \text { is decreasing in time } t \in[0, \infty) \text {. }
$$

Also under the AP flow (4), we have

$$
\text { if } 0<\alpha<1, \quad L^{\alpha-1}(t) \int_{0}^{2 \pi} k^{\alpha-1}(\theta, t) d \theta \quad \text { is increasing in time } t \in[0, \infty) \text {, }
$$

and

$$
\text { if } \alpha=1, \quad \int_{0}^{2 \pi} \log (k(\theta, t) L(t)) d \theta \quad \text { is decreasing in time } t \in[0, \infty) \text {, }
$$

and

$$
\text { if } \alpha>1, \quad L^{\alpha-1}(t) \int_{0}^{2 \pi} k^{\alpha-1}(\theta, t) d \theta \text { is decreasing in time } t \in[0, \infty) \text {. }
$$

Proof. We use (15) and apply (176) in Lemma 4.1 to the LP flow (3) to get

$$
\begin{aligned}
& 2 \pi \int_{0}^{2 \pi} \frac{k^{\alpha}(\theta, t)-\lambda(t)}{k^{2}(\theta, t)} \frac{\partial k}{\partial t}(\theta, t) d \theta \\
& \leq\left(\int_{0}^{2 \pi}\left(k^{\alpha}(\theta, t)-\lambda(t)\right) d \theta\right)^{2}=\left(\frac{d L}{d t}(t)\right)^{2}=0
\end{aligned}
$$

where $\lambda(t)=(2 \pi)^{-1} \int_{0}^{2 \pi} k^{\alpha}(\theta, t)$. In such a case, we will obtain

$$
\frac{d}{d t}\left(\frac{1}{\alpha-1} \int_{0}^{2 \pi} k^{\alpha-1}(\theta, t) d \theta\right) \leq 0
$$

which gives (79) and (80).

On the other hand, apply (177) in Lemma 4.1 to the AP flow (41) to get

$$
\begin{aligned}
& 2 A(t) \int_{0}^{2 \pi} \frac{k^{\alpha}(\theta, t)-\lambda(t)}{k^{2}(\theta, t)} \frac{\partial k}{\partial t}(\theta, t) d \theta \\
& \leq\left(\int_{0}^{2 \pi} \frac{k^{\alpha}(\theta, t)-\lambda(t)}{k(\theta, t)} d \theta\right)^{2}=\left(\frac{d A}{d t}(t)\right)^{2}=0
\end{aligned}
$$


where $\lambda(t)=L^{-1}(t) \int_{0}^{2 \pi} k^{\alpha-1}(\theta, t) d \theta$. The above is the same as

$$
\int_{0}^{2 \pi} k^{\alpha-2}(\theta, t) \frac{\partial k}{\partial t}(\theta, t) d \theta+\frac{L^{\prime}(t)}{L(t)} \int_{0}^{2 \pi} k^{\alpha-1}(\theta, t) d \theta \leq 0, \quad L(t)=\int_{0}^{2 \pi} \frac{1}{k(\theta, t)} d \theta .
$$

For $\alpha=1$, we get (82). For $\alpha>0, \alpha \neq 1$, we get

$$
\frac{1}{\alpha-1} \frac{d}{d t} \log \left(\int_{0}^{2 \pi} k^{\alpha-1}(\theta, t) d \theta\right)+\frac{d}{d t} \log L(t) \leq 0
$$

The result follows.

Remark 4.3 As a comparison, in Gage-Hamilton's curve shortening flow (see [GH]) the integral

$$
\int_{0}^{2 \pi} \log \left[k(\theta, t) \sqrt{\frac{A(t)}{\pi}}\right] d \theta
$$

is decreasing in time, which is called entropy estimate. Now, in the area-preserving curve shortening flow (see [Ga2]) the integral

$$
\int_{0}^{2 \pi} \log [k(\theta, t) L(t)] d \theta
$$

is decreasing in time.

Acknowledgment. We thank the referee for his careful reading of our paper and useful comments and suggestions. We are very grateful to Professor Ben Andrews of the Australian National University. Without consulting him so often, it is unlikely for us to finish this paper. The first author is supported by the National Science Council of Taiwan with grant number 102-2115M-007-012-MY3. The second author is supported by the National Natural Science Foundation of China 11101078, 11171064, and the Natural Science Foundation of Jiangsu Province BK20130596.

\section{References}

[AL] U. Abresch, J. Langer, The normalized curve shortening flow and homothetic solutions, J. Diff. Geom., 23 (1986) 175-196.

[And1] B. Andrews, Evolving convex curves, Cal. Var. \& PDEs, 7 (1998) 315-371.

[And2] B. Andrews, Classification of limiting shapes for isotropic curve flows, J. Amer. Math. Soc., 16 (2003) 443- 459.

[And3] B. Andrews, Volume-preserving anisotropic mean curvature flow, Indiana Univ. Math. J., 50 (2001) 783-827.

[Ang] S. Angenent, On the formation of singularities in the curve shortening flow, J. Diff. Geom., 33 (1991) 601-633.

[B] C. Baker, The mean curvature flow of submanifolds of high codimension, PhD Thesis of the Australian Nat. Univ., 2010. 
[BD] C. Baker, N. Dowson, Area-preserving curvature flow of closed graphs and application to smoothing of PET data, preprint, 2014.

[CLW] X.L. Chao, X.R. Ling, X.L. Wang, On a planar area-preserving curvature flow, Proc. of the AMS, 141 (2013) 1783-1789.

[CZ] K.S. Chou, X.P. Zhu, The Curve Shortening Problem, Chapman \& Hall/CRC, 2001.

[ES] C.-R. Esther, C. Sinestrari, Volume-preserving flow by powers of the mth mean curvature, Cal. Var. \& PDEs, 38 (2010) 441-469.

[G] M.A. Grayson, The heat equation shrinks embedded plane curves to round points, J. Diff. Geom., 26 (1987) 285-314.

[Ga1] M.E. Gage, Curve shortening makes convex curves circular, Invent. Math., 76 (1984) 357-364.

[Ga2] M.E. Gage, On an area-preserving evolution equation for plane curves, Nonlin. Prob. in Geom., Contemp. Math., Amer. Math. Soc., 51 (1985) 51-62.

[Ga3] M. Gage, An isoperimetric inequality with applications to curve shortening, Duke Math. J., 50 (1983) 1225-1229.

[GH] M.E. Gage, R. Hamilton, The heat equation shrinking convex plane curves, J. Diff. Geom., 23 (1986) 69-96.

[H] G. Huisken, The volume preserving mean curvature flow, J. Reine Angew. Math., 382 (1987) 35-48.

[JP] L.S. Jiang, S.L. Pan, On a non-local curve evolution problem in the plane, Comm. Anal. \& Geom., 16 (2008) 1-26.

[LPT1] T.C. Lin, C.C. Poon, D.H. Tsai, Expanding convex immersed closed plane curves, Cal. Var. \& PDEs, 34 (2009) 153-178.

[LPT2] Y.C. Lin, C.C. Poon, D.H. Tsai, Contracting convex immersed closed plane curves with slow speed of curvature, Trans. of the AMS, 364 (2012) 5735-5763.

[LT] Y.C. Lin, D.H. Tsai, Application of Andrews and Green-Osher inequalities to nonlocal flow of convex plane curves, J. of Evo. Eqs., 12 (2012) 833-854.

[M1] J. McCoy, The surface area preserving mean curvature flow, Asian J. Math., 7 (2003) 7-30.

[M2] J. McCoy, Mixed volume preserving curvature flows, Cal. Var. \& PDEs, 24 (2005) 131-154.

[MC] L. Ma, C. Cheng, A non-local area preserving curve flow, Geom. Dedicata, 171 (2014) 231-247.

[MZ] L. Ma, A.Q. Zhu, On a length preserving curve flow, Monatshefte Für Math., 165 (2012) 57-78.

[MPW] Y. Mao, S.L. Pan, Y. Wang, An area-preserving flow for convex closed plane curves, Int. J. Math., 24 (2013) 1350029 (31 pages).

[PT] C.C. Poon, D.H. Tsai, Contracting convex immersed closed plane curves with fast speed of curvature, Comm. Anal. \& Geom., 18 (2010) 23-75. 
[PY] S.L. Pan, J.N. Yang, On a non-local perimeter-preserving curve evolution problem for convex plane curves, Manu. Math., 127 (2008) 469-484.

[PZ] S.L. Pan, H. Zhang, On a curve expanding flow with a nonlocal term, Comm. Contemp. Math., 12 (2010) 815-829.

[S] R. Schneider, Convex Bodies: The Brunn-Minkowski Theory, Cambridge Univ. Press, 1993.

[ST] G. Sapiro, A. Tannenbaum, Area and length preserving geometric invariant scale-spaces, Pattern Anal. \& Machine Intel., IEEE Trans., 17 (1995) 67-72.

[T] K. Tso, Deforming a hypersurface by its Gauss-Kronecker curvature, Comm. Pure Appl. Math., 38 (1985) 867-882.

[U1] J. Urbas, An expansion of convex hypersurfaces, J. Diff. Geom., 33 (1991) 91-125.

[U2] J. Urbas, Convex curves moving homothetically by negative powers of their curvature, Asian J. Math., 3 (1999) 635-658.

[Z] X.P. Zhu, Lectures on Mean Curvature Flows, AMS/IP Studies in Adv. Math., 32 (2002).

Dong-Ho Tsai

Department of Mathematics

National Tsing Hua University

Hsinchu 300, TAIWAN

E-mail:dhtsai@math.nthu.edu.tw

Xiao-Liu Wang

Department of Mathematics

Southeast University

Nanjing 210096, PR CHINA

E-mail: xlwang@seu.edu.cn 